\title{
Soybean Leaf Age and Plant Stage Influence Expression of Resistance to Velvetbean Caterpillar and Fall Armyworm
}

\author{
Bruno Henrique Sardinha de Souza ( $\nabla$ brunosouza@ufla.br) \\ Universidade Federal de Lavras https://orcid.org/0000-0002-2802-2688 \\ Eduardo N. Costa \\ Universidade Federal da Grande Dourados \\ Zulene A. Ribeiro \\ Universidade Estadual Paulista Julio de Mesquita Filho \\ Bruno Perlatti \\ Universidade Federal de Sao Carlos \\ Mara C. P. Cruz \\ Universidade Estadual Paulista Julio de Mesquita Filho \\ Moacir R. Forim \\ Universidade Federal de Sao Carlos \\ Arlindo L. Boiça Júnior \\ Universidade Estadual Paulista Julio de Mesquita Filho \\ Michael J. Stout \\ Louisiana State University
}

\section{Research Article}

Keywords: Flavonoids, Host Plant Resistance, Nutrients, Plant-Insect Interaction, Optimal Defense Theory

Posted Date: April 12th, 2021

DOI: https://doi.org/10.21203/rs.3.rs-355707/v1

License: (c) (1) This work is licensed under a Creative Commons Attribution 4.0 International License. Read Full License

Version of Record: A version of this preprint was published at Chemoecology on August 14th, 2021. See the published version at https://doi.org/10.1007/s00049-021-00360-6. 


\section{Abstract}

Numerous species of herbivorous insects are associated with soybeans, including the specialist velvetbean caterpillar (VBC), Anticarsia gemmatalis, and the generalist fall armyworm (FAW), Spodoptera frugiperda. Expression of plant resistance is influenced by factors intrinsic to host plants, such as leaf age and plant stage, which can differentially affect specialist and generalist insects due to varying levels of plant defense and corresponding insect adaptation. In this study four experiments were carried out to test the hypotheses that levels of antibiosisresistance to VBC and FAW in the resistant genotype PI 227687 and susceptible genotype IGRA RA 626 RR are related to leaf age and plant stage of soybean. Furthermore, the concentrations of nutrients and selected flavonoids were quantified to give insights on possible chemical mechanisms underlying the resistance. As results, development of VBC and FAW were negatively affected when larvae fed leaves of the resistant genotype, older leaves from the lower part of plants, or leaves from reproductive-stage soybeans. The effects were partly different for each insect species, and the generalist FAW was more affected by higher resistance levels in the older leaves of soybean than the specialist VBC. Distribution and concentrations of nutrients and flavonoids in soybean in function of leaf age and plant stage may explain the varying levels of antibiosis-resistance to VBC and FAW. These results can benefit developments of specific protocols for screening resistant soybean genotypes and pest management strategies focused in plant parts and growth stages that insect-resistance levels are lowest.

\section{Key Message}

- Leaf age and plant stage are factors that influence expression of resistance to insects.

- These factors were investigated in soybean genotypes on VBC and FAW development.

- Development of VBC and FAW were negatively affected on the resistant genotype, older leaves, and leaves from reproductive-stage soybeans, and the varying levels of resistance may be explained by flavonoids and nutrients concentrations.

- The results can benefit developments of specific protocols for screening soybean resistant genotypes and pest management strategies.

\section{Introduction}

A diverse assemblage of arthropods is associated with soybeans, Glycine max (L.) Merril. In Brazil, for instance, approximately 40 arthropod species have been reported in soybean fields (Hoffmann-Campo et al. 2003). Among the herbivorous soybean pests, there are species that feed on a narrow range of plants (monophagous/oligophagous or specialists) while others can infest a broad range of plant species (polyphagous or generalists) (Hoffmann-Campo et al. 2012). The velvetbean caterpillar (VBC), Anticarsia gemmatalis Hübner (Lepidoptera: Erebidae), and the fall armyworm (FAW), Spodoptera frugiperda (J. E. Smith) (Lepidoptera: Noctuidae), stand out as defoliating caterpillars that attack soybeans in almost all soybean-producing regions of Brazil (Moscardi et al. 2012). While VBC is an oligophagous species feeding nearly exclusively on leguminous plants (Kogan and Turnipseed 1987), FAW is highly polyphagous and capable of developing on plants from taxonomically diverse families (Barros et al. 2010).

Besides insect specialization on families or species of plants, many insects are adapted to feed on specific parts or phenological stages of their host plants. Acceptance or rejection of plants by herbivorous insects depends on chemical and morphological plant traits (Bernays and Chapman 1994). Many plant species such as soybeans possess diverse types of polyphenolic compounds, with the most abundant group being the flavonoids. Flavonoids are 15-carbon molecules with two aromatic rings connected by a 3-carbon bridge (Crozier et al. 2006). Flavonoid profiles generally differ among families, genera, species, and cultivars of plants (Harborne and Turner 1984). Additionally, concentrations of phenolic compounds can vary throughout plant development (Lynn and Chang 1990). Differences in insect performance and behavior on different plants may be due, at least in part, to the presence and/or concentrations of flavonoids (Simmonds 2001), which are known to mediate soybean antibiosis-resistance (Hoffmann-Campo et al. 2001; Piubelli et al. 2005; Vieira et al. 2016; Bentivenha et al. 2017).

In order to supply insects' needs for growth and reproduction, plants must meet their nutritional requirements (Beck 1972). In general, insects require amino acids, proteins, lipids, sterols, carbohydrates, vitamins, and inorganic nutrients in their diet (Panizzi and Parra 2012), but the nutritional demands vary intra- and interspecifically, and may also vary according to insect age, sex, and function in the population. In some plant-insect systems, expression of plant primary metabolites predicts resistance to pest insects better than does levels of secondary metabolites (Schwachtje and Baldwin 2008; Carmona et al. 2011). Inorganic nutrients in plants, such as nitrogen, phosphorus, and potassium, are associated with plant resistance/susceptibility to herbivorous insects (Poschenrieder et al. 2006). In addition, insect infestation on plants can directly depend on nutrient concentrations in the diverse plant parts and structures (Bastos et al. 2007).

A plant is considered to be resistant when it possesses genes governing the expression of physical, morphological, and/or chemical traits that render the plant less suitable for insect feeding, oviposition, or development. In addition, expression of resistance (antixenosis/antibiosis) can be influenced by diverse factors related to the plant, to the insect, and to the environment (Smith 2005). Studies have demonstrated that factors inherent to the plant, such as leaf age and plant growth stage, substantially influence the expression of antibiosis in soybean genotypes by affecting the development of defoliating caterpillars (Smith and Gilman 1981; Reynolds and Smith 1985; Smith 1985), and of antixenosis by 
altering larval feeding behavior (Boiça Júnior et al. 2015). Understanding the effects of leaf age and plant stage on expression of soybean resistance will contribute to current knowledge on the bioecological interactions among this crop species with specialist and generalist insect herbivores. Additionally, understading the fundamental bases of these complex interactions can facilitate the design of experiments for screening soybean genotypes with insect-resistance traits, and ultimately give insight on how best use plant resistance in integrated pest management strategies.

Based on previous finding that older leaves from the lower part of plants and reproductive-stage soybeans express higher levels of antixenosisresistance to VBC and FAW than younger leaves from the upper part of plants and vegetative-stage soybeans (Boiça Júnior et al. 2015), we tested the hypotheses that levels of antibiosis to these specialist and generalist insects in resistant and susceptible soybean genotypes are related to both leaf age and plant stage. Further, we also measured the concentrations of macronutrients and micronutrients, as well as those of selected flavonoids in soybean leaves to give insights on possible chemical mechanisms underlying the resistance.

\section{Materials And Methods}

\section{Experimental conditions, plant material, and test insects}

Experiments were performed from December 2012 to May 2013 in a greenhouse and laboratory of the Department of Plant Health of the São Paulo State University, School of Agriculture and Veterinarian Sciences, Jaboticabal, state of São Paulo, Brazil. Laboratory bioassays were conducted under environmentally controlled conditions $\left(25 \pm 2^{\circ} \mathrm{C}\right.$ temperature; $70 \pm 10 \%$ relative humidity; $12 \mathrm{~L}: 12 \mathrm{D}$ h photoperiod).

Soybean plants of the resistant genotype PI 227687 and of the susceptible genotype IGRA RA 626 RR were chosen as they were previously screened for resistance to lepidopteran larvae (Souza et al. 2012, Souza et al. 2014) and were reported to vary in the levels of antixenosisresistance in function of leaf age and plant growth stage (Boiça Júnior et al. 2015). Plants were grown in 5-L pots filled with soil (dystrophic red latosol) (Centurion et al. 1995), bovine manure, and sand at 3:1:1 ratio, and kept in a greenhouse under ambient temperature and lighting until used in assays. The plants were watered daily. Larvae of $A$. gemmatalis and $S$. frugiperda were obtained from colonies maintained in the laboratory since September 2011. VBC and FAW larvae were fed on artificial diet (Greene et al. 1976) and the adults on 10\% honey solution.

Four independent experiments were conducted to investigate the effects of leaf age and plant stage on expression of soybean resistance to each insect species, as described below. The first and second experiments compared the development of VBC and FAW fed on younger leaves from the upper part and older leaves from the lower part of plants. The third and fourth experiments compared the insects' development on leaves from soybeans at vegetative and reproductive growth stages. Experiments 1 and 2 were conducted simultaneously using the same batch of soybean plants and under the same environmental conditions, and the same was performed for Experiments 3 and 4 . These standardized conditions allowed to compare and discuss the results regarding the effects of leaf age and plant stage on expression of soybean resistance to the specialist VBC and generalist FAW.

\section{Experiment 1: Effects of soybean leaf age on VBC development}

The first experiment evaluated the influence of leaf age on expression of antibiosis-resistance to VBC. Plants of the resistant and susceptible soybean genotypes were used at the V5 stage (Fehr and Cavines 1977). The "younger leaves" consisted of the first and second trifoliates from the plant apex and the "older leaves" consisted of the third and fourth trifoliates from the same V5 plants. Neonates of VBC were collected from the rearing colony and transferred into 9-cm-diameter Petri dishes lined with moistened filter paper using a fine paintbrush. One larva was placed per dish, which was allowed to feed on either younger or older leaflets of the resistant or susceptible genotypes during the larval stage. Leaflets in the dishes were changed every three days from neonates to third instars, and then replenished daily until pupation.

The experiment was arranged in a completely randomized design, and treatments consisted of a $2 \times 2$ factorial. The four treatments consisted of combinations of two soybean genotypes (PI 227687 and IGRA RA 626 RR) and two leaf ages (younger and older leaves). Five replications were used per treatment, with five individual larvae per Petri dish considered as a replication. Duration of the larval and pupal stages, larval and pupal survival, and larval and pupal weights of VBC were recorded. Larval weights were recorded from 10-d-old larvae and pupal weights in 24h-old pupae.

\section{Experiment 2: Effects of soybean leaf age on FAW development}

The second experiment evaluated the influence of leaf age on expression of antibiosis to FAW. The same treatments, experimental design, number of replicates, and environmental conditions of Experiment 1 were employed, except that larval weights of FAW were recorded in 12 -d-old larvae.

\section{Experiment 3: Effects of soybean plant stage on VBC development}


The third experiment compared the antibiosis-resistance expression to VBC at vegetative and reproductive soybean plant stages. V5-stage plants were used for the vegetative stage, while R3-R4-stage plants were used for the reproductive stage (Fehr and Cavines 1977). For this experiment leaves were collected from the upper part (younger leaves) of soybean plants at both growth stages and then offered to VBC larvae in Petri dishes. For vegetative-stage plants, the first/second trifoliates from the plant apex were used, whereas the first/second/third trifoliates from the apex were collected from reproductive-stage plants.

The experiment was arranged in a completely randomized design, and the four treatments consisted of a $2 \times 2$ factorial consisting of combinations of two soybean genotypes (PI 227687 and IGRA RA 626 RR) and two plant stages (vegetative and reproductive). Five replications were used per treatment, with five individual larvae per Petri dish considered as a replication. The same biological parameters of VBC in Experiment 1 were recorded in this experiment.

\section{Experiment 4: Effects of soybean plant stage on FAW development}

The fourth experiment compared the antibiosis-resistance expression to FAW at vegetative and reproductive growth stages. The same growth stages of soybean were used for the vegetative (V5) and reproductive (R3-R4) stages as in Experiment 3. However, the leaf age/position within plants that were used in the bioassay with FAW was different from that of VBC; here the third/fourth trifoliates were collected from vegetativestage plants and the fourth/fifth/sixth trifoliates from reproductive-stage plants and then offered to FAW larvae in Petri dishes. Different methodologies between VBC and FAW bioassays were used because of the varying results for each insect species; a clearer distinction in the levels of antixenosis-resistance in reproductive-stage soybeans to FAW was obtained in previous evaluation using older leaves collected from the lower part of plants relative to younger leaves from the upper part of plants (Boiça Júnior et al. 2015).

Other conditions of this experiment such as treatments, experimental design, and replication were similar to those of Experiment 3 , except that larval weights of FAW were recorded in 12-d-old larvae.

\section{Concentrations of nutrients and flavonoids in younger and older leaves of soybeans at vegetative and reproductive stages}

To give insights on possible chemical mechanisms underlying the antibiosis-resistance to VBC and FAW in soybean in function of leaf age and plant stage as observed in the bioassays, plants of both genotypes were further cultivated to collect leaves for analysis of nutrients and flavonoids. For this, resistant and susceptible soybeans were grown in two small field plots consisting of four 5-m-long rows of plants spaced $0.5 \mathrm{~m}$ apart, with stands of 10-12 plants per row meter. Soil type in the field plots was the same as that used for potted plants, and was fertilized once with bovine manure before seeds sowing. Field-grown plants were watered by drip irrigation 2-3 times a week. Preventive insecticide and fungicide applications were applied to soybean plants to prevent damage by biotic stressors and minimize unintended alterations in plant biochemistry due to induced defense responses.

When plants reached the V5 stage, 30 plants were randomly selected for each genotype. "Younger leaves" were collected from the first/second trifoliates (upper part) from the plant apex, and "older leaves" were collected from the third/fourth trifoliates (lower part) of the same plants. The leaves were stored in paper bags and taken to the laboratory. When the set of plants of the other field plot reached the R3-R4 reproductive stage, 30 plants of each genotype were randomly selected, and "younger leaves" were collected from the first/second/third trifoliates (upper part) from the plant apex, and "older leaves" from the fourth/fifth/sixth trifoliates of the same plants.

Analysis of nutrients was performed in the Department of Soils and Fertilizers of the School of Agriculture and Veterinarian Sciences. In the laboratory, leaves of each soybean leaf-age and plant-stage categories collected from the 30 plants were pooled together for each genotype in order to reduce potential between-plant variability. The leaves were rinsed with demineralized water $+0.5 \%$ neutral detergent solution, and rinsed consecutively four times with demineralized water. Next, they were oven-dried at $60^{\circ} \mathrm{C}$ for $48 \mathrm{~h}$ and milled to a fine powder through a rotary-knife mill (Thomas Wiley Mill, model Te 650, Swedesboro, NJ, USA) with $1 \mathrm{~mm}$ mesh size. The macronutrients N, P, K, Ca, Mg, and S, and the micronutrients B, Cu, Fe, Mn, and Zn were quantified following the methodology of Miyazawa et al. (1999). A single sample consisting of leaflets pooled together from 30 plants of each treatment combination was analyzed in triplicate.

Quantitative analysis of flavonoids was performed in the Department of Chemistry of the Federal University of São Carlos, in São Carlos, state of São Paulo, Brazil. The flavonoids rutin, isoquercitrin, daidzin, daidzein, hesperidin, naringin, naringenin, and hesperidin in younger and older leaves of the resistant and susceptible soybeans at vegetative and reproductive stages were quantified using high performance liquid chromatography - mass spectrometry (HPLC-MS) following the methodology developed by Perlatti et al. (2016).

\section{Statistical analysis}

Data of biological parameters of VBC and FAW were checked for normality of residuals and homogeneity of variance using the KolmogorovSmirnov and Levene tests, respectively. FAW larval and pupal weights data were not normally distributed and were square root-transformed to meet assumptions of the analysis of variance (ANOVA). Data were subjected to two-way ANOVA, with genotype, leaf age/plant stage

Page $4 / 16$ 
(depending on the experiment), and their interactions as main effects. Means of treatments were separated by Tukey honestly significant difference (HSD) test $(a=0.05)$. Statistical analysis was performed in Statistica v.7 (Statsoft 2004).

\section{Results}

\section{Experiment 1: Effects of soybean leaf age on VBC development}

There was no significant effect of genotype on VBC larval stage duration (Table 1). Larvae that were fed older leaves took longer to develop to pupae than did larvae fed younger leaves. The effects of genotype and leaf age on VBC larval stage duration were independent. Significant differences in VBC larval survival (Table 1) were found between genotypes and leaf ages. Larvae fed leaves of the resistant genotype or older leaves of soybeans had lower survival than larvae fed leaves of the susceptible genotype or younger leaves. There was no significant interaction of genotype and leaf age on the larval survival. Larval weights of VBC were not significantly affected by genotype (Table 1), but were affected by leaf age; larvae that were fed older leaves weighed $41 \%$ less than larvae fed younger leaves of soybeans. There was no significant interaction of genotype and leaf age on VBC larval weight.

Table 1

Mean ( \pm SE) duration (d) of larval and pupal stages, larval and pupal survival (\%), and weight (mg) of larvae and pupae of $A$. gemmatalis fed on younger leaves and older leaves of resistant (PI 227687) and susceptible (IGRA RA 626 RR) soybeans

\begin{tabular}{|c|c|c|c|c|c|c|c|c|c|}
\hline \multirow[t]{2}{*}{ Genotypes } & \multicolumn{3}{|c|}{ Duration of larval stage (d) } & \multicolumn{3}{|c|}{ Larval survival (\%) } & \multicolumn{3}{|c|}{ Larval weight (mg) } \\
\hline & $\begin{array}{l}\text { Younger } \\
\text { leaves }\end{array}$ & $\begin{array}{l}\text { Older } \\
\text { leaves }\end{array}$ & Mean & $\begin{array}{l}\text { Younger } \\
\text { leaves }\end{array}$ & $\begin{array}{l}\text { Older } \\
\text { leaves }\end{array}$ & Mean & $\begin{array}{l}\text { Younger } \\
\text { leaves }\end{array}$ & $\begin{array}{l}\text { Older } \\
\text { leaves }\end{array}$ & Mean \\
\hline PI 227687 & $\begin{array}{l}15.41 \pm \\
0.39 \mathrm{aA}\end{array}$ & $\begin{array}{l}17.75 \pm \\
0.82 \mathrm{aA}\end{array}$ & $\begin{array}{l}16.16 \pm \\
0.43 a\end{array}$ & $\begin{array}{l}68.00 \pm \\
9.52 \mathrm{aA}\end{array}$ & $\begin{array}{l}32.00 \pm \\
9.52 \mathrm{aA}\end{array}$ & $\begin{array}{l}50.00 \pm \\
7.14 a\end{array}$ & $\begin{array}{l}238.89 \pm \\
12.85 \mathrm{aA}\end{array}$ & $\begin{array}{l}108.33 \pm \\
23.78 \mathrm{aA}\end{array}$ & $\begin{array}{l}186.67 \pm \\
16.91 a\end{array}$ \\
\hline $\begin{array}{l}\text { IGRA RA } \\
626 \text { RR }\end{array}$ & $\begin{array}{l}14.96 \pm \\
0.22 \mathrm{aA}\end{array}$ & $\begin{array}{l}16.60 \pm \\
0.38 a A\end{array}$ & $\begin{array}{l}15.59 \pm \\
0.23 a\end{array}$ & $\begin{array}{l}96.00 \pm \\
4.00 \mathrm{aA}\end{array}$ & $\begin{array}{l}60.00 \pm \\
10.00 \mathrm{aA}\end{array}$ & $\begin{array}{l}78.00 \pm \\
5.91 b\end{array}$ & $\begin{array}{l}253.75 \pm \\
9.88 a A\end{array}$ & $\begin{array}{l}169.50 \pm \\
19.41 \mathrm{aA}\end{array}$ & $\begin{array}{l}215.46 \pm \\
11.98 a\end{array}$ \\
\hline Mean & $\begin{array}{l}15.15 \pm \\
0.21 \mathrm{~A}\end{array}$ & $\begin{array}{l}17.00 \pm \\
0.38 \mathrm{~B}\end{array}$ & & $\begin{array}{l}82.00 \pm \\
5.49 \mathrm{~B}\end{array}$ & $\begin{array}{l}46.00 \pm \\
7.12 \mathrm{~A}\end{array}$ & & $\begin{array}{l}247.38 \pm \\
7.85 B\end{array}$ & $\begin{array}{l}146.56 \pm \\
15.63 \mathrm{~A}\end{array}$ & \\
\hline $\begin{array}{l}\text { Genotype } \\
\text { (G) }\end{array}$ & $\begin{array}{l}F_{1,16}= \\
2.18\end{array}$ & $\begin{array}{l}P= \\
0.1448\end{array}$ & ns & $\begin{array}{l}F_{1,16}= \\
14.00\end{array}$ & $P=0.0018$ & $\star \star$ & $\begin{array}{l}F_{1,16}= \\
3.20\end{array}$ & $P=0.0779$ & ns \\
\hline $\begin{array}{l}\text { Plant } \\
\text { factor }(F)\end{array}$ & $\begin{array}{l}F_{1,16}= \\
22.31\end{array}$ & $\begin{array}{l}P< \\
0.0001\end{array}$ & $\star \star \star$ & $\begin{array}{l}F_{1,16}= \\
23.14\end{array}$ & $P=0.0002$ & $\star \star \star$ & $\begin{array}{l}F_{1,16}= \\
39.98\end{array}$ & $P<0.0001$ & $\star \star \star$ \\
\hline$G \times F$ & $\begin{array}{l}F_{1,16}= \\
1.76\end{array}$ & $\begin{array}{l}P= \\
0.1898\end{array}$ & ns & $\begin{array}{l}F_{1,16}= \\
0.00\end{array}$ & $P=0.9975$ & ns & $\begin{array}{l}F_{1,16}= \\
3.37\end{array}$ & $P=0.0707$ & ns \\
\hline \multirow[t]{2}{*}{ Genotypes } & \multicolumn{3}{|c|}{ Duration of pupal stage (d) } & \multicolumn{3}{|c|}{ Pupal survival (\%) } & \multicolumn{3}{|c|}{ Pupal weight (mg) } \\
\hline & $\begin{array}{l}\text { Younger } \\
\text { leaves }\end{array}$ & $\begin{array}{l}\text { Older } \\
\text { leaves }\end{array}$ & Mean & $\begin{array}{l}\text { Younger } \\
\text { leaves }\end{array}$ & $\begin{array}{l}\text { Older } \\
\text { leaves }\end{array}$ & Mean & $\begin{array}{l}\text { Younger } \\
\text { leaves }\end{array}$ & $\begin{array}{l}\text { Older } \\
\text { leaves }\end{array}$ & Mean \\
\hline PI 227687 & $\begin{array}{l}8.94 \pm \\
0.23 \mathrm{aA}\end{array}$ & $\begin{array}{l}9.14 \pm \\
0.14 \mathrm{aA}\end{array}$ & $\begin{array}{l}9.00 \pm \\
0.17 a\end{array}$ & $\begin{array}{l}100.00 \pm \\
0.00 \mathrm{aA}\end{array}$ & $\begin{array}{l}87.50 \pm \\
12.50 \mathrm{aA}\end{array}$ & $\begin{array}{l}96.00 \pm \\
4.00 a\end{array}$ & $\begin{array}{l}208.82 \pm \\
8.30 \mathrm{aA}\end{array}$ & $\begin{array}{l}202.50 \pm \\
12.65 \mathrm{aA}\end{array}$ & $\begin{array}{l}206.80 \pm \\
6.83 a\end{array}$ \\
\hline $\begin{array}{l}\text { IGRA RA } \\
626 \text { RR }\end{array}$ & $\begin{array}{l}9.00 \pm \\
0.18 \mathrm{aA}\end{array}$ & $\begin{array}{l}8.83 \pm \\
0.21 \mathrm{aA}\end{array}$ & $\begin{array}{l}8.94 \pm \\
0.14 a\end{array}$ & $\begin{array}{l}100.00 \pm \\
0.00 \mathrm{aA}\end{array}$ & $\begin{array}{l}80.00 \pm \\
10.69 \mathrm{aA}\end{array}$ & $\begin{array}{l}92.11 \pm \\
4.43 a\end{array}$ & $\begin{array}{l}211.67 \pm \\
9.35 \mathrm{aA}\end{array}$ & $\begin{array}{l}197.86 \pm \\
10.56 \mathrm{aA}\end{array}$ & $\begin{array}{l}206.58 \pm \\
7.07 a\end{array}$ \\
\hline Mean & $\begin{array}{l}8.98 \pm \\
0.14 \AA\end{array}$ & $\begin{array}{l}8.95 \pm \\
0.14 \AA\end{array}$ & & $\begin{array}{l}100.00 \pm \\
0.00 \mathrm{~B}\end{array}$ & $\begin{array}{l}82.61 \pm \\
8.08 \mathrm{~A}\end{array}$ & & $\begin{array}{l}210.49 \pm \\
6.39 A\end{array}$ & $\begin{array}{l}199.55 \pm \\
7.97 \mathrm{~A}\end{array}$ & \\
\hline $\begin{array}{l}\text { Genotype } \\
\text { (G) }\end{array}$ & $\begin{array}{l}F_{1,16}= \\
0.07\end{array}$ & $\begin{array}{l}P= \\
0.7949\end{array}$ & ns & $\begin{array}{l}F_{1,16}= \\
0.41\end{array}$ & $P=0.5234$ & ns & $\begin{array}{l}F_{1,16}= \\
0.00\end{array}$ & $P=0.9832$ & ns \\
\hline $\begin{array}{l}\text { Plant } \\
\text { factor (F) }\end{array}$ & $\begin{array}{l}F_{1,16}= \\
0.01\end{array}$ & $\begin{array}{l}P= \\
0.9048\end{array}$ & ns & $\begin{array}{l}F_{1,16}= \\
7.96\end{array}$ & $P=0.0065$ & ** & $\begin{array}{l}F_{1,16}= \\
1.04\end{array}$ & $P=0.3131$ & ns \\
\hline$G \times F$ & $\begin{array}{l}F_{1,16}= \\
0.60\end{array}$ & $\begin{array}{l}P= \\
0.4407\end{array}$ & ns & $\begin{array}{l}F_{1,16}= \\
0.12\end{array}$ & $P=0.7339$ & ns & $\begin{array}{l}F_{1,16}= \\
0.11\end{array}$ & $P=0.7364$ & ns \\
\hline
\end{tabular}

Pupal duration of VBC (Table 1) was not influenced by soybean genotype, leaf age, or their interaction. Survival of pupae whose larvae were fed older leaves was significantly lower than survival of pupae in younger leaves (Table 1). The main effects of soybean genotype and the 
interactive effect of genotype and leaf age did not influence the pupal survival. Weights of VBC pupae (Table 1) were not influenced either by soybean genotype, leaf age, or by the genotype $\times$ leaf age interaction.

\section{Experiment 2: Effects of soybean leaf age on FAW development}

For FAW, there were no significant effects of genotype, leaf age, or genotype $\times$ leaf age on the duration of the larval stage (Table 2). Soybean genotype, leaf age, and the genotype $\times$ leaf age interaction significantly affected FAW larval survival (Table 2 ). The survival of larvae that were fed leaves of the resistant genotype and older leaves of soybeans was reduced relative to the survival in the susceptible genotype and younger leaves; significant differences for larval survival between genotypes were found only in the older leaves. Weights of FAW larvae were influenced by genotype and leaf age. Larvae fed leaves of the resistant genotype exhibited weights $12 \%$ lower than weights of larvae fed leaves of the susceptible genotype, whereas larvae fed older leaves were $45 \%$ lighter than larvae fed younger leaves. The effect of genotype was dependent on that of leaf age. Larvae of FAW fed older leaves of both genotypes weighed significantly less than larvae fed younger foliage; however, significant differences in larval weights between genotypes occurred only in older leaves.

Table 2

Mean ( \pm SE) duration (d) of larval and pupal stages, larval and pupal survival (\%), and weight (mg) of larvae and pupae of $S$. frugiperda fed on younger leaves and older leaves of resistant (PI 227687) and susceptible (IGRA RA 626 RR) soybeans

\begin{tabular}{|c|c|c|c|c|c|c|c|c|c|}
\hline \multirow[t]{2}{*}{ Genotypes } & \multicolumn{3}{|c|}{ Duration of larval stage (d) } & \multicolumn{3}{|c|}{ Larval survival (\%) } & \multicolumn{3}{|c|}{ Larval weight (mg) } \\
\hline & $\begin{array}{l}\text { Younger } \\
\text { leaves }\end{array}$ & $\begin{array}{l}\text { Older } \\
\text { leaves }\end{array}$ & Mean & $\begin{array}{l}\text { Younger } \\
\text { leaves }\end{array}$ & $\begin{array}{l}\text { Older } \\
\text { leaves }\end{array}$ & Mean & $\begin{array}{l}\text { Younger } \\
\text { leaves }\end{array}$ & $\begin{array}{l}\text { Older } \\
\text { leaves }\end{array}$ & Mean \\
\hline PI 227687 & $\begin{array}{l}18.15 \pm \\
0.62 a A\end{array}$ & $\begin{array}{l}20.67 \pm \\
1.45 \mathrm{aA}\end{array}$ & $\begin{array}{l}18.48 \pm \\
0.59 a\end{array}$ & $\begin{array}{l}80.00 \pm \\
8.16 \mathrm{aB}\end{array}$ & $\begin{array}{l}12.00 \pm \\
6.63 a A\end{array}$ & $\begin{array}{l}46.00 \pm \\
7.12 a\end{array}$ & $\begin{array}{l}261.25 \pm \\
21.04 \mathrm{aB}\end{array}$ & $\begin{array}{l}43.57 \pm \\
14.32 \mathrm{aA}\end{array}$ & $\begin{array}{l}181.05 \pm \\
22.30 a\end{array}$ \\
\hline $\begin{array}{l}\text { IGRA RA } \\
626 \text { RR }\end{array}$ & $\begin{array}{l}17.74 \pm \\
0.25 \mathrm{aA}\end{array}$ & $\begin{array}{l}18.78 \pm \\
0.51 \mathrm{aA}\end{array}$ & $\begin{array}{l}18.20 \pm \\
0.25 a\end{array}$ & $\begin{array}{l}92.00 \pm \\
5.54 \mathrm{aA}\end{array}$ & $\begin{array}{l}72.00 \pm \\
9.17 \mathrm{bA}\end{array}$ & $\begin{array}{l}82.00 \pm \\
5.49 b\end{array}$ & $\begin{array}{l}252.00 \pm \\
14.29 \mathrm{aB}\end{array}$ & $\begin{array}{l}157.39 \pm \\
18.84 \mathrm{bA}\end{array}$ & $\begin{array}{l}206.67 \pm \\
13.78 b\end{array}$ \\
\hline Mean & $\begin{array}{l}17.93 \pm \\
0.32 A\end{array}$ & $\begin{array}{l}19.05 \pm \\
0.50 \mathrm{~A}\end{array}$ & & $\begin{array}{l}86.00 \pm \\
4.96 \mathrm{~B}\end{array}$ & $\begin{array}{l}42.00 \pm \\
7.05 \mathrm{~A}\end{array}$ & & $\begin{array}{l}256.53 \pm \\
12.50 \mathrm{~B}\end{array}$ & $\begin{array}{l}114.32 \pm \\
16.22 \mathrm{~A}\end{array}$ & \\
\hline $\begin{array}{l}\text { Genotype } \\
\text { (G) }\end{array}$ & $\begin{array}{l}F_{1,16}= \\
0.26\end{array}$ & $\begin{array}{l}P= \\
0.6121\end{array}$ & ns & $\begin{array}{l}F_{1,16}= \\
22.35\end{array}$ & $P<0.0001$ & $\star \star \star$ & $\begin{array}{l}F_{1,16}= \\
6.02\end{array}$ & $P=0.0163$ & * \\
\hline $\begin{array}{l}\text { Plant } \\
\text { factor (F) }\end{array}$ & $\begin{array}{l}F_{1,16}= \\
3.88\end{array}$ & $\begin{array}{l}P= \\
0.0536\end{array}$ & ns & $\begin{array}{l}F_{1,16}= \\
33.38\end{array}$ & $P<0.0001$ & $\star \star \star ~$ & $\begin{array}{l}F_{1,16}= \\
61.07\end{array}$ & $P<0.0001$ & $\star \star \star$ \\
\hline$G \times F$ & $\begin{array}{l}F_{1,16}= \\
2.16\end{array}$ & $\begin{array}{l}P= \\
0.1473\end{array}$ & ns & $\begin{array}{l}F_{1,16}= \\
9.93\end{array}$ & $P=0.0062$ & $\star \star$ & $\begin{array}{l}F_{1,16}= \\
20.36\end{array}$ & $P<0.0001$ & $\star \star \star$ \\
\hline \multirow[t]{2}{*}{ Genotypes } & \multicolumn{3}{|c|}{ Duration of pupal stage (d) } & \multicolumn{3}{|c|}{ Pupal survival (\%) } & \multicolumn{3}{|c|}{ Pupal weight (mg) } \\
\hline & $\begin{array}{l}\text { Younger } \\
\text { leaves }\end{array}$ & $\begin{array}{l}\text { Older } \\
\text { leaves }\end{array}$ & Mean & $\begin{array}{l}\text { Younger } \\
\text { leaves }\end{array}$ & $\begin{array}{l}\text { Older } \\
\text { leaves }\end{array}$ & Mean & $\begin{array}{l}\text { Younger } \\
\text { leaves }\end{array}$ & $\begin{array}{l}\text { Older } \\
\text { leaves }\end{array}$ & Mean \\
\hline PI 227687 & $\begin{array}{l}9.26 \pm \\
0.17 \mathrm{aA}\end{array}$ & $\begin{array}{l}9.00 \pm \\
0.00 \mathrm{aA}\end{array}$ & $\begin{array}{l}9.23 \pm \\
0.15 a\end{array}$ & $\begin{array}{l}95.00 \pm \\
5.00 \mathrm{aA}\end{array}$ & $\begin{array}{l}100.00 \pm \\
0.00 \mathrm{aA}\end{array}$ & $\begin{array}{l}95.65 \pm \\
4.35 a\end{array}$ & $\begin{array}{l}213.50 \pm \\
4.17 \mathrm{aA}\end{array}$ & $\begin{array}{l}176.67 \pm \\
13.47 a A\end{array}$ & $\begin{array}{l}208.70 \pm \\
4.72 a\end{array}$ \\
\hline $\begin{array}{l}\text { IGRA RA } \\
626 \text { RR }\end{array}$ & $\begin{array}{l}8.96 \pm \\
0.13 \mathrm{aA}\end{array}$ & $\begin{array}{l}9.82 \pm \\
0.40 \mathrm{aA}\end{array}$ & $\begin{array}{l}9.33 \pm \\
0.20 a\end{array}$ & $\begin{array}{l}100.00 \pm \\
0.00 \mathrm{aA}\end{array}$ & $\begin{array}{l}94.44 \pm \\
5.56 \mathrm{aA}\end{array}$ & $\begin{array}{l}97.56 \pm \\
2.44 a\end{array}$ & $\begin{array}{l}223.04 \pm \\
5.81 \mathrm{aA}\end{array}$ & $\begin{array}{l}196.67 \pm \\
6.10 \mathrm{aA}\end{array}$ & $\begin{array}{l}211.46 \pm \\
4.63 a\end{array}$ \\
\hline Mean & $\begin{array}{l}9.10 \pm \\
0.11 \mathrm{~A}\end{array}$ & $\begin{array}{l}9.70 \pm \\
0.39 \mathrm{~B}\end{array}$ & & $\begin{array}{l}97.67 \pm \\
2.33 \mathrm{~A}\end{array}$ & $\begin{array}{l}95.24 \pm \\
4.76 \mathrm{~A}\end{array}$ & & $\begin{array}{l}218.61 \pm \\
3.68 \mathrm{~B}\end{array}$ & $\begin{array}{l}193.81 \pm \\
5.66 \mathrm{~A}\end{array}$ & \\
\hline $\begin{array}{l}\text { Genotype } \\
\text { (G) }\end{array}$ & $\begin{array}{l}F_{1,16}= \\
0.13\end{array}$ & $\begin{array}{l}P= \\
0.7254\end{array}$ & ns & $\begin{array}{l}F_{1,16}= \\
0.17\end{array}$ & $P=0.6816$ & ns & $\begin{array}{l}F_{1,16}= \\
0.19\end{array}$ & $P=0.6634$ & ns \\
\hline $\begin{array}{l}\text { Plant } \\
\text { factor (F) }\end{array}$ & $\begin{array}{l}F_{1,16}= \\
4.55\end{array}$ & $\begin{array}{l}P= \\
0.0371\end{array}$ & * & $\begin{array}{l}F_{1,16}= \\
0.27\end{array}$ & $P=0.6084$ & ns & $\begin{array}{l}F_{1,16}= \\
14.70\end{array}$ & $P=0.0003$ & $\star \star \star$ \\
\hline$G \times F$ & $\begin{array}{l}F_{1,16}= \\
2.36\end{array}$ & $\begin{array}{l}P= \\
0.1296\end{array}$ & ns & $\begin{array}{l}F_{1,16}= \\
0.93\end{array}$ & $P=0.3392$ & ns & $\begin{array}{l}F_{1,16}= \\
3.20\end{array}$ & $P=0.0786$ & ns \\
\hline
\end{tabular}

Duration of the pupal stage was longer in FAW whose larvae were fed older leaves than in those reared on younger leaves (Table 2). Pupal stage duration was not influenced either by soybean genotype or by the genotype $\times$ leaf age interaction. Survival of FAW pupae (Table 2) was 
not affected by genotype, leaf age, or by their interaction. FAW fed older leaves of soybeans yielded lighter pupae than in younger leaves. The effects of genotype and genotype $\times$ leaf age interaction were not significant.

\section{Experiment 3: Effects of soybean plant stage on VBC development}

Genotype and plant stage of soybeans significantly affected the larval stage duration of VBC (Table 3). Larval duration was longer on the resistant genotype than on the susceptible genotype. VBC larvae that were fed leaves from reproductive-stage soybeans showed increased duration of the larval stage compared to larvae fed vegetative-stage soybeans. The effects of genotype and plant stage were independent. Survival of VBC larvae was not affected by soybean genotype, plant stage, or the interaction of these factors (Table 3). Weights of VBC larvae (Table 3) were significantly affected by genotype and plant stage, but there was no interaction between these factors. Larvae that were fed the resistant genotype had body weights $20 \%$ lower than weights of larvae fed the susceptible genotype, whereas larvae fed leaves from reproductive-stage plants had body weights $46 \%$ lower than larvae fed vegetative-stage plants.

Table 3

Mean ( \pm SE) duration (d) of larval and pupal stages, larval and pupal survival (\%), and weight (mg) of larvae and pupae of $A$. gemmatalis fed on leaves from resistant (PI 227687) and susceptible (IGRA RA 626 RR) soybeans at vegetative and reproductive growth stages

\begin{tabular}{|c|c|c|c|c|c|c|c|c|c|}
\hline \multirow[t]{2}{*}{ Genotypes } & \multicolumn{3}{|c|}{ Duration of larval stage (d) } & \multicolumn{3}{|c|}{ Larval survival (\%) } & \multicolumn{3}{|c|}{ Larval weight (mg) } \\
\hline & Vegetative & Reproductive & Mean & Vegetative & Reproductive & Mean & Vegetative & Reproductive & Mean \\
\hline PI 227687 & $\begin{array}{l}14.70 \pm \\
0.32 \mathrm{aA}\end{array}$ & $\begin{array}{l}16.53 \pm \\
0.32 \mathrm{aA}\end{array}$ & $\begin{array}{l}15.60 \\
\pm \\
0.27 b\end{array}$ & $\begin{array}{l}80.00 \pm \\
8.16 \mathrm{aA}\end{array}$ & $\begin{array}{l}76.00 \pm \\
8.72 \mathrm{aA}\end{array}$ & $\begin{array}{l}78.00 \\
\pm \\
5.92 a\end{array}$ & $\begin{array}{l}173.33 \pm \\
12.37 \mathrm{aA}\end{array}$ & $\begin{array}{l}106.25 \pm \\
9.21 \mathrm{aA}\end{array}$ & $\begin{array}{r}139.79 \\
\pm 9.14 \mathrm{a}\end{array}$ \\
\hline $\begin{array}{l}\text { IGRA RA } \\
626 \text { RR }\end{array}$ & $\begin{array}{l}13.76 \pm \\
0.19 \mathrm{aA}\end{array}$ & $\begin{array}{l}16.30 \pm \\
0.40 \mathrm{aA}\end{array}$ & $\begin{array}{l}14.89 \\
\pm \\
0.28 a\end{array}$ & $\begin{array}{l}100.00 \pm \\
0.00 \mathrm{aA}\end{array}$ & $\begin{array}{l}80.00 \pm \\
8.16 \mathrm{aA}\end{array}$ & $\begin{array}{l}90.00 \\
\pm \\
4.29 a\end{array}$ & $\begin{array}{l}225.60 \pm \\
10.09 a A\end{array}$ & $\begin{array}{l}110.95 \pm \\
12.90 \mathrm{aA}\end{array}$ & $\begin{array}{l}173.26 \\
\pm \\
11.67 b\end{array}$ \\
\hline Mean & $\begin{array}{l}14.18 \pm \\
0.19 A\end{array}$ & $\begin{array}{l}16.41 \pm \\
0.25 \mathrm{~B}\end{array}$ & & $\begin{array}{l}90.00 \pm \\
4.29 \mathrm{~A}\end{array}$ & $\begin{array}{l}78.00 \pm \\
5.92 \mathrm{~A}\end{array}$ & & $\begin{array}{l}200.00 \pm \\
8.72 B\end{array}$ & $\begin{array}{l}108.44 \pm \\
7.69 \mathrm{~A}\end{array}$ & \\
\hline $\begin{array}{l}\text { Genotype } \\
\text { (G) }\end{array}$ & $\begin{array}{l}F_{1,16}= \\
5.34\end{array}$ & $P=0.0234$ & * & $\begin{array}{l}F_{1,16}= \\
4.24\end{array}$ & $P=0.0563$ & ns & $\begin{array}{l}F_{1,16}= \\
8.87\end{array}$ & $P=0.0037$ & $\star \star$ \\
\hline $\begin{array}{l}\text { Plant } \\
\text { factor (F) }\end{array}$ & $\begin{array}{l}F_{1,16}= \\
54.20\end{array}$ & $P<0.0001$ & $\star \star \star ~$ & $\begin{array}{l}F_{1,16}= \\
4.24\end{array}$ & $P=0.0563$ & ns & $\begin{array}{l}F_{1,16}= \\
66.26\end{array}$ & $P<0.0001$ & $\star \star \star$ \\
\hline$G \times F$ & $\begin{array}{l}F_{1,16}= \\
0.03\end{array}$ & $P=0.8669$ & ns & $\begin{array}{l}F_{1,16}= \\
1.88\end{array}$ & $P=0.1890$ & ns & $\begin{array}{l}F_{1,16}= \\
2.49\end{array}$ & $P=0.1182$ & ns \\
\hline \multirow[t]{2}{*}{ Genotypes } & \multicolumn{3}{|c|}{ Duration of pupal stage $(\mathrm{d})$} & \multicolumn{3}{|c|}{ Pupal survival (\%) } & \multicolumn{3}{|c|}{ Pupal weight (mg) } \\
\hline & Vegetative & Reproductive & Mean & Vegetative & Reproductive & Mean & Vegetative & Reproductive & Mean \\
\hline PI 227687 & $\begin{array}{l}9.17 \pm \\
0.12 \mathrm{aA}\end{array}$ & $\begin{array}{l}9.84 \pm \\
0.16 \mathrm{aA}\end{array}$ & $\begin{array}{l}9.51 \\
\pm \\
0.11 \mathrm{~b}\end{array}$ & $\begin{array}{l}90.00 \pm \\
6.88 \mathrm{aA}\end{array}$ & $\begin{array}{l}100.00 \pm \\
0.00 \mathrm{aA}\end{array}$ & $\begin{array}{l}94.87 \\
\pm \\
3.58 \mathrm{a}\end{array}$ & $\begin{array}{l}163.50 \pm \\
5.68 \mathrm{aA}\end{array}$ & $\begin{array}{l}213.16 \pm \\
6.84 a A\end{array}$ & $\begin{array}{l}187.69 \\
\pm 5.90 a\end{array}$ \\
\hline $\begin{array}{l}\text { IGRA RA } \\
626 \text { RR }\end{array}$ & $\begin{array}{l}9.04 \pm \\
0.12 \mathrm{aA}\end{array}$ & $\begin{array}{l}9.38 \pm \\
0.21 \mathrm{aA}\end{array}$ & $\begin{array}{l}9.17 \\
\pm \\
0.11 \mathrm{a}\end{array}$ & $\begin{array}{l}92.00 \pm \\
5.54 \mathrm{aA}\end{array}$ & $\begin{array}{l}80.00 \pm \\
9.18 \mathrm{aA}\end{array}$ & $\begin{array}{l}86.67 \\
\pm \\
5.12 a\end{array}$ & $\begin{array}{l}181.60 \pm \\
6.29 a A\end{array}$ & $\begin{array}{l}235.00 \pm \\
6.68 \mathrm{aA}\end{array}$ & $\begin{array}{l}205.33 \\
\pm 6.04 \mathrm{~b}\end{array}$ \\
\hline Mean & $\begin{array}{l}9.10 \pm \\
0.08 \mathrm{~A}\end{array}$ & $9.66 \pm 0.13 \mathrm{~B}$ & & $\begin{array}{l}91.11 \pm \\
4.29 \mathrm{~A}\end{array}$ & $\begin{array}{l}89.74 \pm \\
4.92 \mathrm{~A}\end{array}$ & & $\begin{array}{l}173.56 \pm \\
4.46 \mathrm{~A}\end{array}$ & $\begin{array}{l}224.36 \pm \\
5.04 \mathrm{~B}\end{array}$ & \\
\hline $\begin{array}{l}\text { Genotype } \\
\text { (G) }\end{array}$ & $\begin{array}{l}F_{1,16}= \\
5.60\end{array}$ & $P=0.0208$ & * & $\begin{array}{l}F_{1,16}= \\
1.65\end{array}$ & $P=0.2033$ & ns & $\begin{array}{l}F_{1,16}= \\
7.51\end{array}$ & $P=0.0076$ & $\star \star$ \\
\hline $\begin{array}{l}\text { Plant } \\
\text { factor (F) }\end{array}$ & $\begin{array}{l}F_{1,16}= \\
14.31\end{array}$ & $P=0.0003$ & $\star \star \star$ & $\begin{array}{l}F_{1,16}= \\
0.05\end{array}$ & $P=0.8313$ & ns & $\begin{array}{l}F_{1,16}= \\
62.25\end{array}$ & $P<0.0001$ & $\star \star \star$ \\
\hline$G \times F$ & $\begin{array}{l}F_{1,16}= \\
0.00\end{array}$ & $P=0.9975$ & ns & $\begin{array}{l}F_{1,16}= \\
2.97\end{array}$ & $P=0.0889$ & ns & $\begin{array}{l}F_{1,16}= \\
2.06\end{array}$ & $P=0.1549$ & ns \\
\hline
\end{tabular}

Duration of VBC pupal stage (Table 3) was influenced by soybean genotype and plant stage. The duration was extended when VBC was fed leaves from the resistant genotype and reproductive-stage plants. The genotype $\times$ plant stage interaction on the pupal stage duration was not 
significant. Survival of VBC pupae (Table 3) was not affected by genotype, plant stage or by their interaction. Larvae that were fed leaves of the resistant genotype produced pupae with lower weights than those fed the susceptible genotype (Table 3). Larvae that fed leaves from reproductive-stage soybeans produced pupae with greater weights than pupae developed on vegetative-stage soybeans. The effects of genotype and plant stage on FAW pupal weight did not interact.

\section{Experiment 4: Effects of soybean plant stage on FAW development}

The larval duration of FAW significantly differed between genotypes and plant stages. Larvae fed leaves of the resistant genotype or reproductive-stage plants developed more slowly compared to larvae fed leaves of the susceptible genotype or vegetative-stage soybeans. The effects of genotype and plant stage were independent. FAW larval survival was lower in the resistant genotype relative to the susceptible genotype (Table 4). Feeding of FAW on leaves from soybeans at different growth stages did not influence larval survival. There were no interactive effects of genotype and plant stage on FAW larval survival. Larvae fed leaves of the resistant genotype or leaves from reproductivestage plants resulted in weights $40 \%$ lower than weights of larvae fed leaves of the susceptible genotype or vegetative-stage plants. No significant genotype $\times$ plant stage interaction was found for the larval weight (Table 4).

Table 4

Mean ( \pm SE) duration (d) of larval and pupal stages, larval and pupal survival (\%), and weight (mg) of larvae and pupae of $S$. frugiperda fed on leaves from resistant (PI 227687) and susceptible (IGRA RA 626 RR) soybeans at vegetative and reproductive growth stages

\begin{tabular}{|c|c|c|c|c|c|c|c|c|c|}
\hline \multirow[t]{2}{*}{ Genotypes } & \multicolumn{3}{|c|}{ Duration of larval stage (d) } & \multicolumn{3}{|c|}{ Larval survival (\%) } & \multicolumn{3}{|c|}{ Larval weight (mg) } \\
\hline & Vegetative & Reproductive & Mean & Vegetative & Reproductive & Mean & Vegetative & Reproductive & Mean \\
\hline PI 227687 & $\begin{array}{l}19.87 \pm \\
0.27 \mathrm{aA}\end{array}$ & $\begin{array}{l}23.69 \pm \\
0.80 \mathrm{aA}\end{array}$ & $\begin{array}{l}21.84 \\
\pm \\
0.55 b\end{array}$ & $\begin{array}{l}60.00 \pm \\
10.00 \mathrm{aA}\end{array}$ & $\begin{array}{l}64.00 \pm \\
9.80 \mathrm{aA}\end{array}$ & $\begin{array}{l}62.00 \\
\pm \\
6.93 a\end{array}$ & $\begin{array}{l}200.00 \pm \\
14.98 \mathrm{aA}\end{array}$ & $\begin{array}{l}95.26 \pm \\
11.31 \mathrm{aA}\end{array}$ & $\begin{array}{l}151.46 \\
\pm \\
12.58 a\end{array}$ \\
\hline $\begin{array}{l}\text { IGRA RA } \\
626 \text { RR }\end{array}$ & $\begin{array}{l}17.91 \pm \\
0.23 \mathrm{aA}\end{array}$ & $\begin{array}{l}19.56 \pm \\
0.36 \mathrm{aA}\end{array}$ & $\begin{array}{l}18.68 \\
\pm \\
0.24 a\end{array}$ & $\begin{array}{l}92.00 \pm \\
5.54 \mathrm{aA}\end{array}$ & $\begin{array}{l}72.00 \pm \\
9.17 \mathrm{aA}\end{array}$ & $\begin{array}{l}82.00 \\
\pm \\
5.49 \mathrm{~b}\end{array}$ & $\begin{array}{l}323.33 \pm \\
18.62 \mathrm{aA}\end{array}$ & $\begin{array}{l}195.42 \pm \\
19.38 \mathrm{aA}\end{array}$ & $\begin{array}{l}259.38 \\
\pm \\
16.30 b\end{array}$ \\
\hline Mean & $\begin{array}{l}18.68 \pm \\
0.23 \mathrm{~A}\end{array}$ & $\begin{array}{l}21.50 \pm \\
0.55 \mathrm{~B}\end{array}$ & & $\begin{array}{l}76.00 \pm \\
6.10 \mathrm{~A}\end{array}$ & $\begin{array}{l}68.00 \pm \\
6.66 \mathrm{~A}\end{array}$ & & $\begin{array}{l}264.35 \pm \\
15.15 B\end{array}$ & $\begin{array}{l}151.16 \pm \\
14.10 A\end{array}$ & \\
\hline $\begin{array}{l}\text { Genotype } \\
\text { (G) }\end{array}$ & $\begin{array}{l}F_{1,16}= \\
52.36\end{array}$ & $P<0.0001$ & $\star \star \star ~$ & $\begin{array}{l}F_{1,16}= \\
5.79\end{array}$ & $P=0.0286$ & $\star$ & $\begin{array}{l}F_{1,16}= \\
34.16\end{array}$ & $P<0.0001$ & $\star \star \star$ \\
\hline $\begin{array}{l}\text { Plant } \\
\text { factor (F) }\end{array}$ & $\begin{array}{l}F_{1,16}= \\
41.09\end{array}$ & $P<0.0001$ & $\star \star \star$ & $\begin{array}{l}F_{1,16}= \\
1.79\end{array}$ & $P=0.2001$ & ns & $\begin{array}{l}F_{1,16}= \\
43.55\end{array}$ & $P<0.0001$ & $\star \star \star$ \\
\hline$G \times F$ & $\begin{array}{l}F_{1,16}= \\
0.00\end{array}$ & $P=0.9975$ & ns & $\begin{array}{l}F_{1,16}= \\
3.50\end{array}$ & $P=0.0798$ & ns & $\begin{array}{l}F_{1,16}= \\
2.94\end{array}$ & $P=0.0903$ & ns \\
\hline \multirow[t]{2}{*}{ Genotypes } & \multicolumn{3}{|c|}{ Duration of pupal stage $(\mathrm{d})$} & \multicolumn{3}{|c|}{ Pupal survival (\%) } & \multicolumn{3}{|c|}{ Pupal weight (mg) } \\
\hline & Vegetative & Reproductive & Mean & Vegetative & Reproductive & Mean & Vegetative & Reproductive & Mean \\
\hline PI 227687 & $\begin{array}{l}9.54 \pm \\
0.14 \mathrm{aA}\end{array}$ & $\begin{array}{l}9.40 \pm \\
0.27 \mathrm{aA}\end{array}$ & $\begin{array}{l}9.48 \\
\pm \\
0.14 a\end{array}$ & $\begin{array}{l}86.67 \pm \\
9.09 a A\end{array}$ & $\begin{array}{l}62.25 \pm \\
12.50 \mathrm{aA}\end{array}$ & $\begin{array}{l}74.19 \\
\pm \\
7.99 a\end{array}$ & $\begin{array}{l}176.00 \pm \\
9.15 \mathrm{aA}\end{array}$ & $\begin{array}{l}221.25 \pm \\
13.60 \mathrm{aA}\end{array}$ & $\begin{array}{r}199.36 \\
\pm 9.11 \mathrm{a}\end{array}$ \\
\hline $\begin{array}{l}\text { IGRA RA } \\
626 \text { RR }\end{array}$ & $\begin{array}{l}9.18 \pm \\
0.13 \mathrm{aA}\end{array}$ & $\begin{array}{l}9.29 \pm \\
0.14 \mathrm{aA}\end{array}$ & $\begin{array}{l}9.24 \\
\pm \\
0.09 a\end{array}$ & $\begin{array}{l}82.61 \pm \\
8.08 \mathrm{aA}\end{array}$ & $\begin{array}{l}94.44 \pm \\
5.56 \mathrm{aA}\end{array}$ & $\begin{array}{l}87.80 \\
\pm \\
5.17 a\end{array}$ & $\begin{array}{l}216.36 \pm \\
9.60 \mathrm{aA}\end{array}$ & $\begin{array}{l}235.00 \pm \\
10.86 a A\end{array}$ & $\begin{array}{l}224.75 \\
\pm 7.24 b\end{array}$ \\
\hline Mean & $\begin{array}{l}9.33 \pm \\
0.10 A\end{array}$ & $9.33 \pm 0.13 \mathrm{~A}$ & & $\begin{array}{l}84.21 \pm \\
5.99 A\end{array}$ & $\begin{array}{l}79.41 \pm \\
7.04 \mathrm{~A}\end{array}$ & & $\begin{array}{l}200.00 \pm \\
7.46 \mathrm{~A}\end{array}$ & $\begin{array}{l}228.53 \pm \\
8.54 \mathrm{~B}\end{array}$ & \\
\hline $\begin{array}{l}\text { Genotype } \\
\text { (G) }\end{array}$ & $\begin{array}{l}F_{1,16}= \\
2.19\end{array}$ & $P=0.1451$ & ns & $\begin{array}{l}F_{1,16}= \\
2.29\end{array}$ & $P=0.1353$ & ns & $\begin{array}{l}F_{1,16}= \\
5.27\end{array}$ & $P=0.0249$ & * \\
\hline $\begin{array}{l}\text { Plant } \\
\text { factor (F) }\end{array}$ & $\begin{array}{l}F_{1,16}= \\
0.00\end{array}$ & $P=0.9975$ & ns & $\begin{array}{l}F_{1,16}= \\
0.29\end{array}$ & $P=0.5928$ & ns & $\begin{array}{l}F_{1,16}= \\
6.74\end{array}$ & $P=0.0116$ & * \\
\hline$G \times F$ & $\begin{array}{l}F_{1,16}= \\
0.61\end{array}$ & $P=0.4382$ & ns & $\begin{array}{l}F_{1,16}= \\
3.86\end{array}$ & $P=0.0536$ & ns & $\begin{array}{l}F_{1,16}= \\
2.28\end{array}$ & $P=0.1360$ & ns \\
\hline
\end{tabular}


There were no significant effects of soybean genotype, plant stage or genotype $\times$ plant stage interaction on FAW pupal duration (Table 4). Likewise, pupal survival (Table 4) was not influenced by genotype, plant stage, or by their interaction. Pupae of FAW reared on the resistant genotype showed an $11 \%$ reduction in weights compared to pupae reared on the susceptible genotype. FAW pupae originating from reproductive-stage plants showed increased weights compared to pupae from vegetative-stage plants. The interaction of genotype and plant stage was not significant.

\section{Concentrations of nutrients and flavonoids in younger and older leaves of soybeans at vegetative and reproductive stages}

In vegetative-stage plants, higher concentrations of macronutrients and micronutrients were overall recorded in the susceptible genotype than in the resistant genotype (Table 5). As an exception, higher values of the micronutrients $\mathrm{B}, \mathrm{Cu}$, and Fe were observed in the resistant genotype. All macronutrients but $\mathrm{Ca}$ and $\mathrm{Mg}$ showed higher values in younger leaves than in older leaves; while Ca showed higher concentrations in the older leaves, Mg was evenly distributed between younger and older foliage. For the micronutrients, younger leaves had slightly higher concentrations of $\mathrm{Cu}$ than older leaves of both soybean genotypes. Conversely, concentrations of Fe, Mn, and Zn were higher in the older leaves. There was no distribution pattern of $\mathrm{B}$ between younger and older leaves of vegetative-stage plants in the soybean genotypes. 
Table 5

Concentrations of macronutrients $\left(\mathrm{g} \mathrm{kg}^{-1}\right)$ and micronutrients $\left(\mathrm{mg} \mathrm{kg}^{-1}\right)$ in younger and older leaves of resistant (PI 227687) and susceptible (IGRA RA 626 RR) soybeans at vegetative and reproductive stages

\begin{tabular}{|c|c|c|c|c|c|c|}
\hline \multirow[t]{2}{*}{ Nutrients } & \multicolumn{3}{|l|}{ PI 227687} & \multicolumn{3}{|l|}{ IGRA RA 626 RR } \\
\hline & Younger leaves & Older leaves & Mean & Younger leaves & Older leaves & Mean \\
\hline \multicolumn{7}{|c|}{ Vegetative stage } \\
\hline \multicolumn{7}{|c|}{ Macronutrients } \\
\hline $\mathrm{N}$ & 42.0 & 35.0 & 38.5 & 49.0 & 40.0 & 44.5 \\
\hline$P$ & 2.9 & 2.3 & 2.6 & 3.3 & 2.3 & 2.8 \\
\hline $\mathrm{K}$ & 17.0 & 14.0 & 15.5 & 16.0 & 14.0 & 15.0 \\
\hline $\mathrm{Ca}$ & 6.9 & 9.0 & 8.0 & 9.5 & 11.2 & 10.4 \\
\hline $\mathrm{Mg}$ & 3.8 & 4.0 & 3.9 & 4.6 & 4.5 & 4.6 \\
\hline $\mathrm{S}$ & 2.5 & 2.0 & 2.3 & 2.9 & 2.3 & 2.6 \\
\hline \multicolumn{7}{|c|}{ Micronutrients } \\
\hline$B$ & 43.0 & 47.0 & 45.0 & 46.0 & 35.0 & 40.5 \\
\hline $\mathrm{Cu}$ & 6.0 & 5.0 & 5.5 & 4.0 & 3.0 & 3.5 \\
\hline $\mathrm{Fe}$ & 207.0 & 353.0 & 280.0 & 144.0 & 269.0 & 206.5 \\
\hline $\mathrm{Mn}$ & 116.0 & 157.0 & 136.5 & 189.0 & 236.0 & 212.5 \\
\hline $\mathrm{Zn}$ & 31.0 & 32.0 & 31.5 & 38.0 & 46.0 & 42.0 \\
\hline \multicolumn{7}{|c|}{ Reproductive stage } \\
\hline \multicolumn{7}{|c|}{ Macronutrients } \\
\hline $\mathrm{N}$ & 34.0 & 29.0 & 31.5 & 45.0 & 42.0 & 43.5 \\
\hline$P$ & 4.1 & 3.9 & 4.0 & 5.3 & 4.2 & 4.8 \\
\hline K & 13.0 & 13.0 & 13.0 & 11.0 & 12.0 & 11.5 \\
\hline $\mathrm{Ca}$ & 19.0 & 22.0 & 20.5 & 27.0 & 25.0 & 26.0 \\
\hline $\mathrm{Mg}$ & 3.0 & 3.0 & 3.0 & 4.0 & 3.0 & 3.5 \\
\hline S & 1.4 & 1.3 & 1.4 & 1.8 & 1.7 & 1.8 \\
\hline \multicolumn{7}{|c|}{ Micronutrients } \\
\hline B & 45.0 & 46.0 & 45.5 & 35.0 & 45.0 & 40.0 \\
\hline $\mathrm{Cu}$ & 7.0 & 8.0 & 7.5 & 6.0 & 8.0 & 7.0 \\
\hline $\mathrm{Fe}$ & 230.0 & 218.0 & 224.0 & 171.0 & 365.0 & 268.0 \\
\hline $\mathrm{Mn}$ & 71.0 & 77.0 & 74.0 & 95.0 & 86.0 & 90.5 \\
\hline $\mathrm{Zn}$ & 47.0 & 59.0 & 53.0 & 39.0 & 71.0 & 55.0 \\
\hline
\end{tabular}

At the reproductive stage, higher concentrations of nutrients were also found in the susceptible genotype than in the resistant genotype (Table 5). Contrary to this trend, the macronutrient $\mathrm{K}$ and the micronutrients $\mathrm{B}$ and $\mathrm{Cu}$ had higher values in the resistant genotype.

Concentrations of $\mathrm{N}, \mathrm{K}, \mathrm{Mg}, \mathrm{S}$, and $\mathrm{Mn}$ were lower at the reproductive stage than at the vegetative stage, whereas $\mathrm{P}, \mathrm{Ca}, \mathrm{Cu}$, and $\mathrm{Zn}$ showed increased values as plants matured. The micronutrient Fe had reduced values from the vegetative to the reproductive stage only in the resistant genotype, whereas in the susceptible genotype the concentrations increased. The macronutrients $\mathrm{N}$ and $\mathrm{P}$ and the micronutrient $\mathrm{S}$ had higher values in the younger leaves than in the older leaves of reproductive-stage soybeans. On the other hand, contents of $\mathrm{B}$, $\mathrm{Cu}$, and $\mathrm{Zn}$ were higher in the older leaves relative to the younger leaves. Concentrations of $\mathrm{K}$ and $\mathrm{Mg}$ did not differ between leaf-age categories. Finally, Ca, Fe, and $\mathrm{Mn}$ exhibited no distribution pattern between younger and older leaves in soybean genotypes at the reproductive stage. 
Of the eight selected flavonoids assessed, only rutin, isoquercitrin, daidzin, daidzein, and hesperidin were identified and quantified in the leaves of soybeans (Table 6). Naringin, naringenin, and quercetin were not detected by the HPLC-MS analysis. Therefore, these three flavonoids are not presented in the results.

Table 6

Concentrations of flavonoids $\left(\mu \mathrm{g} \mathrm{g}^{-1}\right)$ in younger and older leaves of resistant (PI 227687) and susceptible (IGRA RA 626 RR) soybeans at vegetative and reproductive stages.

\begin{tabular}{|c|c|c|c|c|c|c|}
\hline \multirow[t]{2}{*}{ Flavonoids } & \multicolumn{3}{|l|}{ PI 227687} & \multicolumn{3}{|l|}{ IGRA RA 626 RR } \\
\hline & Younger leaves & Older leaves & Mean & Younger leaves & Older leaves & Mean \\
\hline \multicolumn{7}{|c|}{ Vegetative stage } \\
\hline Rutin & 3304.14 & 3267.20 & 3285.67 & 840.74 & 828.43 & 834.59 \\
\hline Isoquercitrin & 72.19 & 109.52 & 90.86 & 54.27 & 94.05 & 74.16 \\
\hline Daidzin & 8.73 & 49.63 & 29.18 & 17.12 & 36.53 & 26.83 \\
\hline Daidzein & -a & 10.45 & 10.45 & - & 8.24 & 8.24 \\
\hline Hesperidin & 12.03 & 15.62 & 13.83 & - & 5.50 & 5.50 \\
\hline Total & 3397.09 & 3452.42 & 3424.76 & 912.13 & 972.75 & 942.44 \\
\hline \multicolumn{7}{|c|}{ Reproductive stage } \\
\hline Rutin & 3710.39 & 3156.41 & 3433.40 & 763.18 & 606.83 & 685.01 \\
\hline Isoquercitrin & 113.60 & 127.36 & 120.48 & 75.18 & 58.86 & 67.02 \\
\hline Daidzin & 160.98 & 119.48 & 140.23 & 162.71 & 36.66 & 99.69 \\
\hline Daidzein & 36.77 & 39.23 & 38.00 & 24.72 & 4.35 & 14.54 \\
\hline Hesperidin & 30.25 & 40.55 & 35.40 & - & 1.00 & 1.00 \\
\hline Total & 4051.99 & 3483.03 & 3767.51 & 1025.79 & 707.70 & 866.75 \\
\hline
\end{tabular}

In vegetative-stage plants, rutin had the highest concentrations among the quantified flavonoids (Table 6), exhibiting values many orders of magnitude greater than those of isoquercitrin, daidzin, hesperidin, and daidzein in the resistant and susceptible genotypes. All flavonoids showed higher concentrations in the resistant genotype; this was especially true for rutin, which showed concentration four times higher in the resistant genotype than in the susceptible genotype. Selected flavonoids exhibited higher contents in the older leaves than in the younger leaves, except for rutin. Daidzin was found only in the older leaves in both soybean genotypes. Hesperidin was found in both younger and older leaves of the resistant genotype, but only in the older leaves of the susceptible genotype. Considering the total flavonoids content, the resistant genotype possessed $>3.5$-fold higher concentration than the susceptible genotype in vegetative-stage plants.

Concentrations of flavonoids overall increased from the vegetative to the reproductive stage of soybeans (Table 6). In reproductive-stage plants, rutin also was the most abundant flavonoid, with concentrations in the resistant genotype on average 5-fold greater than in the susceptible genotype. Unlike the vegetative stage, daidzein was quantified in the younger leaves of soybean genotypes at the reproductive stage. Hesperidin was not quantified in younger leaves of the susceptible genotype at the reproductive stage, likewise in vegetative-stage plants. Taken together, plants of the resistant genotype showed nearly 4.5-fold higher total flavonoids concentrations than the susceptible genotype at reproductive stage.

\section{Discussion}

In the present study four independent experiments were conducted with VBC and FAW and resistant and susceptible genotypes to test the hypotheses that leaf age and plant stage affect the expression of antibiosis-resistance in soybean. The effects of soybean genotype and both leaf age and plant stage on VBC development were independent, as was the effect of genotype and plant stage for FAW; larvae that were fed leaves of the resistant genotype, leaves from the lower part of plants, and leaves from reproductive-stage soybeans had the development negatively affected compared to larvae fed leaves of the susceptible genotype, leaves from the upper part of plants, and leaves from vegetativestage soybeans, respectively. On the other hand, soybean genotype effects were dependent on leaf age for FAW, in that only the older leaves of the resistant genotype impaired the insect's development, as evidenced by the lower larval survival and weight on this treatment.

Page $11 / 16$ 
The varying levels of antibiosis-resistance in PI 227687 to VBC and FAW as a function of leaf age and plant stage are in agreement with previous studies performed with the soybean looper Chrysodeixis includens (Walker) (Lepidoptera: Noctuidae) (Smith and Gilman 1981; Reynolds and Smith 1985; Smith 1985). In addition, Boiça Júnior et al. (2015) evaluated the influence of insect- and plant-related factors on soybean antixenosis-resistance to VBC and FAW, and concluded that VBC consumed less on older leaves from the lower part of plants and from reproductive-stage plants; conversely, FAW did not display preference toward younger leaves from the upper part or older leaves from the lower part of plants, but consumed less foliage from reproductive-stage plants. Despite the similar results obtained by these studies, none of them assessed the concentrations of primary and secondary metabolites in soybean to explain the varying levels of resistance in function of leaf age/plant stage. The results obtained here and in the study of Boiça Júnior et al. (2015) for the differential responses of VBC and FAW on the older leaves from the lower part of resistant soybean plants indicate that effects of both antixenosis- and antibiosis-resistance are overlapped, as previously discussed by Stout (2013). This information coupled with the knowledge that leaf age and plant stage influence the magnitude of resistance expression in soybean can benefit the design of specific protocols of high-throughput screening assays for insect-resistance traits (Boiça Júnior et al. 2015; Eduardo et al. 2020).

Differences in effects of genotype and leaf age/plant stage on the development of each insect species in this study were likely due to differences in their feeding behavior and adaptation to soybeans. Larvae of VBC are oligophagous and feed basically on leguminous plants (Kogan and Turnipseed 1987), while larvae of FAW are polyphagous and develop well on plants of taxonomically disparate families (Barros et al. 2010). The response spectrum of insects' gustatory receptors depends on the presence and content of diverse types of allelochemicals within the insects' host-plant range (Smith 2005), and in generalist (polyphagous) insects they display a broader response spectrum than that in specialists (monophagous/oligophagous) (Visser 1983). Based on data of the present study it is suggested that the more specialist an insect is on a host plant, the slighter will be the impact of narrow variation in plants allelochemicals and nutrients contents on its development, and less clear will be the differences in insect-resistance levels between genotypes of the same plant species. This fact coupled with differential expression of allelochemical-detoxifying enzymes by the insects (Bosch and Welte 2017) may explain why FAW was more affected by the higher antibiosis levels in older leaves from the lower part of resistant soybean plants than was VBC. Research using resistant and susceptible soybean genotypes should investigate if these results correlate with patterns of VBC and FAW infestations within plants and across phenological stages in the field.

Macronutrients and micronutrients displayed different distribution patterns between soybean plant parts and growth stages, and their distribution is in line with mobility in the phloem. Some nutrients are highly or moderately mobile in the phloem depending on their functions as metabolic or structural constituents, and concentrate in the youngest and growing leaves of the plant apex (Marschner 1995). Among the nutrients with higher contents in the younger leaves in the current study, N, P, and K are highly mobile, and S and Cu are moderately mobile (Sfredo 2008). Conversely, the micronutrients Ca and Fe were more abundant in the older leaves, and these nutrients have low and intermediate mobility in the phloem, respectively (Sfredo 2008); in addition, Ca cannot be transported after being deposited in a given plant structure (Raven et al. 1996).

The susceptible genotype IGRA RA 626 RR exhibited higher concentrations of most macronutrients than the resistant genotype PI 227687, especially in the younger leaves of vegetative-stage plants, with few exceptions. Despite the nutrients analysis being performed using leaves collected from plants in a parallel experiment from the larvae bioassays, data suggest there may be a positive correlation between better development of VBC and FAW and increased concentrations of macronutrients. Some of them participate in the synthesis of compounds that are fundamental for insect nutrition, such as proteins. Among the macronutrients, $\mathrm{N}$ is an important component of plant proteins and is indispensable in diets of insects (Panizzi and Parra 2012) as it is required for their growth and reproduction (Elden and Kenworthy 1994). Excess $\mathrm{N}$ can influence plant susceptibility through reduction of lignin content, making plant structures softer for feeding of chewing insects (Salim and Saxena 1991). Sulfur is also a constituent of plant proteins (Sfredo 2008); P is required for nucleic acid formation and protein synthesis (Huberty and Denno 2006); and K is fundamental for protein and starch production (Spann and Schumann 2010).

The resistant genotype showed higher concentrations of $\mathrm{Cu}$ and $\mathrm{B}$ relative to the susceptible genotype at both plant growth stages. The role of $\mathrm{Cu}$ in plants is associated with activation of defensive enzymes, such as polyphenol oxidase (Sfredo 2008), peroxidase, catalase (Taiz and Zeiger 2010), and superoxide dismutase (Poschenrieder et al. 2006). Concentrations of Cu are directly associated with enhanced activities of polyphenol oxidase (Mourato et al. 2009), a Cu-containing enzyme that catalyzes the conversion of phenolics to quinones, which play an important role in plant resistance to insects (Bhonwong et al. 2009). Also, plants deficient in Cu build up concentrations of soluble carbohydrates, have impaired synthesis of defensive compounds, and reduced lignification, leading to reduced resistance to fungal diseases (Spann and Schumann 2010). Boron is used for synthesis of flavonoids and other polyphenolic compounds that contribute to plant resistance to insects and pathogens; $B$ deficiency leads to sugar and amino acid accumulation in the leaves and stems, which may render plants more susceptible to insect attack (Spann and Schumann 2010). Therefore, this preliminary data on Cu and B concentrations suggest these micronutrients may be positively correlated with higher levels of antibiosis-resistance to VBC and FAW, and could be candidates for quantification in future screening assays of soybean genotypes for insect-resistance traits.

Page $12 / 16$ 
The flavonoids rutin, isoquercitrin, daidzin, daidzein, and hesperidin were found at different concentrations in soybean genotypes, plant parts and growth stages. Flavonoids concentrations were higher in the resistant genotype, older leaves from the lower part of plants, and reproductive-stage plants. Flavonoids are polyphenolic compounds that are present in many plant species (Harborne 1967), and are reported to have an important role in plant defense against insects (Harborne and Williams 2000; Simmonds 2001; Simmonds 2003). The difference in total flavonoids concentration between soybean genotypes was in the order of magnitude of 3.5 fold greater for the resistant genotype in vegetative-stage plants, and increased to 4.5 fold in the reproductive stage. Although the bioassays with VBC and FAW and the flavonoids analysis were performed with leaves collected from plants in different experiments, the insects' development were impaired on the resistant genotype, older leaves from the lower part of plants, and reproductive-stage plants, which were also the treatments that exhibited higher flavonoids concentrations. It is important to mention that the methodology used herein for flavonoids extraction and quantification was previously validated (Perlatti et al. 2016). Results of experiments with soybeans and other insect species are also in agreement with the hypothesis that higher flavonoids concentrations are correlated with increased resistance levels (Isman and Duffey 1983; Hoffmann-Campo et al. 2001; Piubelli et al. 2005; Hoffmann-Campo et al. 2006; Vieira et al. 2016; Bentivenha et al. 2017).

Although the methodology used in this study for leaf collection did not allow for statistical analysis of individual flavonoids and nutrients concentrations, pooling various leaves together within each soybean genotype-leaf age/plant stage combination certainly decreased data variability that naturally occur between plant individuals. Quantification of these primary and secondary compounds was performed to give insights on potential chemical mechanisms underlying the differences in levels of antibiosis-resistance to VBC and FAW. According to the chemical analysis, it is suggested that younger leaves from the upper part of plants or from vegetative-stage soybeans are less defended and more nutritious, hence more suitable for VBC and FAW development. An in-depth investigation with greater replication should analyze the highlighted nutrients and flavonoids to pinpoint the compounds that potentially have a role in antibiosis; if confirmed, they could be further used as reliable proxies of the chemical mechanisms, contributing to high-throughput phenotyping of soybean genotypes for insect-resistance. Moreover, as leaf collection is routinely practiced by farmers in the field to analyze the need for plants fertilization, it could be possible to optimize the sampling method using younger and older leaves from plants at vegetative and reproductive stages during soybean crop development for assessments of those proxies of plant resistance to correlate with insect population densities; this practice could aid in the decision making of pest control and application of fertilizers that contribute to increasing insect-resistance levels in the plants.

Various theories have been put forward to explain the distribution patterns of defense compounds in different plant parts and structures and the influence on ecology and evolution of plant-insect interactions. The optimal defense theory is one of them, which predicts that plants express defense compounds at highest levels in tissues and organs that contribute to plant fitness to the greatest extent. Younger tissues are suggested to be more valuable in terms of fitness for plants than older tissues; hence, younger tissues are expected to possess higher concentrations of defense compounds than older tissues according to the optimal defense theory (Mckey 1979). Several defense compounds in different plant species were reported to have a within-plant distribution in a fashion following the optimal defense theory (Meldau et al. 2012).

Based on flavonoids distribution in the soybean genotypes, at a glance our results seem not to be in agreement with the optimal defense theory since older leaves from the lower part of plants and leaves from reproductive-stage plants possessed higher flavonoids concentrations than younger leaves from the upper part of plants and leaves from vegetative-stage plants. Moreover, higher concentrations of nutrients related with protein synthesis were observed in the younger leaves and vegetative-stage foliage, especially $\mathrm{N}$, which is used as a proxy of plant-tissue fitness value (Moreira et al. 2012). Boiça Júnior et al. (2015) have previously stated that expression of soybean antixenosis-resistance was not in accordance with the optimal defense theory since the younger leaves were more consumed than the older leaves by VBC.

A possible source of inconsistency of results in correlating concentrations of secondary compounds in plant tissues, the fitness value of the plant tissues, and a plausible plant defense theory to explain the negative effects on insects may arise from the lack of methodology standardization to control for constitutive and induced resistance. Here and in studies of Smith and Gilman (1981), Reynolds and Smith (1985), Smith (1985), and Boiça Júnior et al. (2015) that reported better larval development on younger soybean foliage, the experimental plants were not previously injured, hence the observed effects were only constitutive. Freitas et al. (2017) reported that when resistant and susceptible soybean plants were injured in the older leaves from the lower part of plants by Spodoptera cosmioides larvae, only the resistant genotype induced resistance in the younger leaves from the upper part of plants; resistance induction was not observed in the susceptible genotype or in older leaves from the lower part of plants of both genotypes when plants were injured in the younger leaves from the plant apex. Interestingly, the younger foliage was more suitable to larvae feeding and performance before injury was caused by the larvae, and the authors discussed that the pattern of soybean induced resistance followed the optimal defense theory. Therefore, it is essential that both constitutive and inducible responses to insect herbivory be considered when attempting to correlate the distribution of secondary metabolites in plants and theories of plant defense. Because soybean genotypes exhibit great variation in flavonoids concentrations when injured or not by insects (Vieira et al. 2016; Bentivenha et al. 2017), using soybean as a model plant for testing the optimal defense theory could give further insights on its validity, and how induced resistance could be best used into integrated pest management practices.

Page $13 / 16$ 
Although this study is limited to only two genotypes and a narrow range of primary and secondary metabolites that might govern the resistance, the results demonstrated that soybean leaf age and plant stage influence the magnitude of antibiosis-resistance expression to the specialist VBC and the generalist FAW. In addition, the estimation of nutrients and flavonoids contents in distinct leaf age and plant stage of soybeans can give insights on possible chemical mechanisms involved in soybean-insect interactions and benefit the development of more standardized studies on insect-plant biology and host plant resistance. From a practical standpoint, by knowing the spatial and temporal distribution of flavonoids and nutrients in soybeans and the relationship with the preference and performance of insect pests it could be possible to focus pest monitoring and control approaches in plant parts and phenological stages that expression of resistance levels to insects attack are lowest.

\section{Declarations}

Funding Thanks to Coordenação de Aperfeiçoamento de Pessoal de Nível Superior (Coordination for the Improvement of Higher Education Personnel - CAPES, Brazil, Finance Code 001) for funding this research.

Author contribution statement BHSS and ALBJ designed the study. BHSS, ENC, and ZAR conducted the bioassays. BP and MRF analyzed flavonoids. MCPC analyzed nutrients. BHSS analyzed data and wrote the manuscript. ALBJ and MJS revised the manuscript.

Data availability All data are presented in the manuscript.

Ethical approval This work does not contain any study with human participants.

Conflicts of interest The authors declare that they have no conflicts of interest.

Consent to participate This work does not contain any study with human participants.

\section{References}

1. Barros EM, Torres JB, Ruberson JR, Oliveira MD (2010) Development of Spodoptera frugiperda on different hosts and damage to reproductive structures in cotton. Entomol Exp Appl 137:237-245

2. Bastos SC, Galvão JCC, Picanço MC, Pereira PRG, Cecon PR (2007) Nutrient content affecting Spodoptera frugiperda and Dalbulus maidis occurrence in corn. Insect Sci 14:117-123

3. Beck SD (1972) Nutrition, adaptation and environment. In: Rodriguez JG (ed) Insect and mite nutrition: significance and implications in ecology and pest management. North-Holland, Amsterdam, pp 1-6

4. Bentivenha JPF, Canassa VF, Baldin ELL, Borguini MG, Lima GPP, Lourenção AL (2017) Role of the rutin and genistein flavonoids in soybean resistance to Piezodorus guildinii (Hemiptera: Pentatomidae). Arthropod-Plant Interactions. https://doi.org/10.1007/s11829-0179578-5

5. Bernays EA, Chapman RF (1994) Host-selection by phytophagous insects. Chapman \& Hall, New York

6. Boiça Júnior AL, Souza BHS, Costa EN, Ribeiro ZA, Stout MJ (2015) Factors influencing expression of antixenosis in soybean to Anticarsia gemmatalis and Spodoptera frugiperda (Lepidoptera: Noctuidae). J Econ Entomol 108:317-325

7. Bhonwong A, Stout MJ, Attajarusit J, Tantasawat P (2009) Defensive role of tomato polyphenol oxidases against cotton bollworm (Helicoverpa armigera) and beet armyworm (Spodoptera exigua). J Chem Ecol 35:28-38

8. Carmona D, Lajeunesse MJ, Johnson MTJ (2011) Plant traits that predict resistance to herbivores. Funct Ecol 25:358-367

9. Centurion JF, Andrioli I, Marques Júnior J, Marcgiori DC (1995) Características de latossolos roxos desenvolvidos de rochas alcalinas e básicas de Jaboticabal, SP. Sci Agric 52:226-232

10. Crozier A, Jaganath IB, Clifford MN (2006) Phenols, polyphenols and tannins: an overview. In: Crozier A, Clifford MN, Ashihara H (eds) Plant secondary metabolites: occurrence, structure and role in the human diet. Blackwell Publishing, Oxford, pp 1-24

11. Eduardo WI, Boiça Júnior AL, Moraes RFO, Souza BHS, Louvandini H, Barbosa JC (2020). Protocol for assessing soybean antixenosis to Heliothis virescens. Entomol Exp Appl 168:911-927

12. Elden TC, Kenworthy WJ (1994) Foliar nutrient concentrations of insect susceptible and resistant soybean germplasm. Crop Prot 34:695699

13. Fehr WR, Cavines CE (1977) Stages of soybean development. lowa State University of Science and Technology, Ames

14. Greene GL, Leppla NC, Dickerson WA (1976) Velvet bean caterpillar: a rearing procedure and artificial medium. J Econ Entomol 69:487-488

15. Harborne JB (1967) Comparative biochemistry of the flavonoids. Academic Press, London

16. Harborne JB, Turner L (1984) Plant chemosystematics. Academic Press, London

Page 14/16 
17. Hoffmann-Campo CB, Corrêa-Ferreira BS, Moscardi F (2012) Soja: manejo integrado de insetos e outros artrópodes-praga. Embrapa, Brasília

18. Hoffmann-Campo CB, Oliveira LJ, Moscardi F, Gazzoni DL, Corrêa-Ferreira BS, Lorini I, Borges M, Panizzi AR, Sosa-Gómez DR, Corso IA (2003) Integrated pest management in Brazil. In: Maredia KM, Dakouo D, Mota-Sanchez D (eds) Integrated pest management in the global arena. Cabi Publishing, Crowmwell Press, Trowbridge, pp 285-299

19. Hoffmann-Campo CB, Ramos Neto JA, Oliveira MC, Oliveira LJ (2006) Detrimental effect of rutin on Anticarsia gemmatalis. Pesqui Agropecu Bras 41:1453-1459

20. Huberty AF, Denno RF (2006) Consequences of nitrogen and phosphorus limitation for the performance of two planthoppers with divergent life-history strategies. Oecologia 149:444-455

21. Isman MB, Duffey SS (1983) Pharmacokinetics of chlorogenic acid and rutin in larvae of Heliothis zea. J Insect Physiol 29:295-300

22. Kogan M, Turnipseed SG (1987) Ecology and management of soybean arthropods. Annu Rev Entomol 32:507-538

23. Lynn DG, Chang M (1990) Phenolic signals in cohabitation: implications for plant development. Annu Rev Plant Phys 41:497-526

24. McKey D (1979) The distribution of secondary metabolites within plants. In: Rosenthal GA, Jansen DH (eds) Herbivores: their interaction with secondary plant metabolites. Academic Press, New York, pp 55-133

25. Marschner H (1995) Mineral nutrition of higher plants, 2nd edn. Academic, San Diego

26. Meldau S, Erb M, Baldwin LT (2012) Defence on demand: mechanisms behind optimal defence theory. Ann Bot-London. https://doi.org/10.1093/aob/mcs212

27. Miyazawa M, Pavan MA, Muraoka T, Carmo CAFS, Mello MJ (1999) Análises químicas de tecido vegetal. In: Silva FC (ed) Manual de análises químicas de solos, plantas e fertilizantes. Embrapa-SPI, Brasília, pp 171-223

28. Moreira X, Zas R, Sampedro L (2012) Differential allocation of constitutive and induced chemical defenses in pine tree juveniles: a test of the optimal defense theory. PloS One 7: e34006

29. Moscardi, F, Bueno AF, Sosa-Gómez DR, Roggia S, Hoffmann-Campo CB, Pomari AF, Corso IC, Yano SAC (2012) Artrópodes que atacam as folhas da soja. pp 213-334. In Hoffmann-Campo CB, Corrêa-Ferreira BS, Moscardi F (eds.), Soja: manejo integrado de insetos e outros artrópodes-praga. Embrapa, Brasília, Brazil

30. Mourato MP, Martins LL, Campos-Andrada MP (2009) Physiological responses of Lupinus luteus to different copper concentrations. Biol Plantarum 53:105-111

31. Panizzi AR, Parra JRP (2012) Introduction to insect bioecology and nutrition for Integrated Pest Management (IPM). pp 3-12. In Panizzi AR, Parra JRP (eds.), Insect bioecology and nutrition for Integrated Pest Management. CRC Press, Boca Raton, USA

32. Perlatti B, Fernandes JB, Silva MFGF, Ardila JA, Carneiro RL, Souza BHS, Costa EN, Eduardo WI, Boiça Júnior AL, Forim MR (2016) Application of a quantitative HPLC-ESI-MS/MS method for flavonoids in different vegetables matrices. Journal of the Brazilian Chemical Society 27:475-483

33. Piubelli GC, Hoffmann-Campo CB, Moscardi F, Miyakubo SH, Oliveira MCN (2005) Are chemical compounds important for soybean resistance to Anticarsia gemmatalis? J Chem Ecol 31:1509-1525

34. Poschenrieder C, Tolrà R, Barceló J (2006) Can metals defend plants against biotic stress? Trends Plant Sci 11:288-295

35. Raven PH, Evert RF, Eichhorn SE (1996) Biologia vegetal, 5th edn. Guanabara Koogan, Rio de Janeiro

36. Reynolds GW, Smith CM (1985) Effects of leaf position, leaf wounding, and plant age of two soybean genotypes on soybean looper (Lepidoptera: Noctuidae) growth. Environ Entomol 14:475-478

37. Salim M, Saxena MC (1991) Nutritional stresses and varietal resistance in rice: effects on whitebacked planthopper. Crop Sci 31:797-805

38. Schwachtje J, Baldwin IT (2008) Why does herbivore attack reconfigure primary metabolism? Plant Physiol 146:845-851

39. Sfredo JG (2008) Soja no Brasil: calagem, adubação e nutrição animal. Embrapa Soja, Londrina (Documentos, 305)

40. Simmonds MSJ (2003) Flavonoid-insect interactions: recent advances in our knowledge. Phytochemistry 64:21-30

41. Simmonds MSJ (2001) Importance of flavonoids in insect plant-interactions: feeding and oviposition. Phytochemistry 56:245-252

42. Smith CM (1985) Expression, mechanisms and chemistry of resistance in soybean, Glycine max L. (Merr.) to the soybean looper, Pseudoplusia includes (Walker). International Journal of Tropical Insect Science 6:243-248

43. Smith CM (2005) Plant resistance to arthropods: molecular and conventional approaches. Springer, Dordrecht

44. Smith CM, Gilman DF (1981) Comparative resistance of multiple insect-resistant soybean genotypes to the soybean looper. J Econ Entomol 74:400-403

45. Souza BHS, Boiça Júnior AL, Janini JC, Silva AG, Rodrigues NEL (2012) Feeding of Spodoptera eridania (Lepidoptera: Noctuidae) on soybean genotypes. Rev Colomb Entomol 38:215-223

Page $15 / 16$ 
46. Souza BHS, Silva AG, Janini JC, Boiça Júnior AL (2014) Antibiosis in soybean genotypes and the resistance levels to Spodoptera eridania (Cramer) (Lepidoptera: Noctuidae). Neotrop Entomol 43:582-587

47. Spann TM, Schumann AW (2010) Mineral nutrition contributes to plant disease and pest resistance (HS 1181). University of Florida Institute of Food and Agricultural Sciences, Gainesville. http://edis.ifas.ufl.edu/hs1181. Accessed 15 Out 2015

48. Statsoft, Inc (2004) Statistica (data analysis software system), version 7. StatSoft, Inc., Tulsa, USA. http://www.statsoft.com. Accessed 12 Mar 2014

49. Stout MJ (2013) Reevaluating the conceptual framework for applied research on host plant resistance. Insect Sci 20:263-272

50. Taiz L, Zeiger E (2010) Plant physiology, 5th edn. Sinauer Associates, Sunderland

51. van den Bosch TJM, Welte CU (2017) Detoxifying symbionts in agriculturally important pest insects. Microbial biotechnology 10:531-540

52. Vieira SS, Lourenção AL, Graça JP, Janegitz P, Salvador MC, Oliveira MCN, Hoffmann-Campo CB (2016) Biological aspects of Bemisia tabaci biotype B and the chemical causes of resistance in soybean genotypes. Arthropod-Plant Interactions 10:525-534

53. Visser JH (1983) Differential sensory perceptions of plant compounds by insects. In: Hedin PA (ed) Plant resistance to insects. American Chemical Society, Washington, pp 216-243 